\title{
Boletim informativo para prevenção e diagnóstico precoce do câncer de mama
}

\author{
Milena Calado Santana ${ }^{1}$ \\ Julia Raulino de Souza Rippel ${ }^{2}$ \\ Renata Costa Fortes ${ }^{3}$
}

1. Fisioterapeuta Residente do Programa Multiprofissional em Atenção ao Câncer da Escola Superior de Ciências da Saúde - ESCS/FEPECS.

2. Fisioterapeuta Residente do Programa Multiprofissional em Atenção ao Câncer da Escola Superior de Ciências da Saúde - ESCS/FEPECS.

3. Nutricionista, Doutora e Mestra em Nutrição Humana, Coordenadora do Programa Multiprofissional em Atenção ao Câncer da Escola Superior de Ciências da Saúde - ESCS/FEPECS.

\section{RESUMO}

Introdução: $O$ câncer de mama é uma doença multifatorial que se apresenta em grande incidência entre as mulheres brasileiras. A idade é um dos principais fatores de risco, além do uso abusivo de álcool, uso de contraceptivos e histórico de câncer na família. O autoexame da mama também é utilizado como uma forma de prevenção e diagnóstico precoce. A detecção precoce do câncer de mama é importante para reduzir as taxas de mortalidade. Objetivo: O objetivo deste estudo foi elaborar um boletim informativo para a população sobre a importância da prevenção e do diagnóstico precoce de câncer por meio do autoexame das mamas. Métodos: O boletim informativo foi realizado a partir de três etapas: revisão narrativa da literatura, análise do conteúdo e produção prática do boletim. Resultados: Produção do boletim informativo, contendo os principais achados da literatura: definição, aspectos epidemiológicos, fatores de risco, prevenção, autoexame da mama, sinais e sintomas, e diagnóstico. Conclusões: É evidente que o câncer de mama afeta a vida de mulheres brasileiras, sendo que a melhor forma de prevenção é o autoexame. Esse boletim poderá subsidiar a população sobre a importância da prevenção e do diagnóstico precoce por meio do autoexame das mamas.

PALAVRAS CHAVE: Neoplasias da mama, Autoexame, Prevenção de Doenças, Diagnóstico.

\section{Newsletter for prevention and early diagnosis of breast cancer}

\begin{abstract}
Introduction: Breast cancer is a multifactorial disease that appears in great incidence among Brazilian women. Age is one of the main risk factors, in addition to alcohol abuse, use of contraceptives and a family history of cancer. Breast self-examination is also used as a means of prevention and early diagnosis. Early detection of breast cancer is important to reduce the mortality rate. Objective: The aim of this study was to prepare a newsletter on the importance of early warning and diagnosis by early breast self-examination. Methods: The newsletter was produced in three stages: bibliographic research for literature review, content analysis and practical production of the newsletter. Results: Production of the newsletter. Conclusions: It is evident that breast cancer affects the lives of Brazilian women. In the form of prevention or self-examination.
\end{abstract}

KEYWORDS: Breast neoplasms, Self-Examination, Disease Prevention, Diagnosis. 


\section{INTRODUÇÃO}

O câncer de mama é uma doença causada pela multiplicação desordenada de células da mama, esse processo gera células anormais que se multiplicam, formando um tumor maligno. Esse tipo de câncer também acomete homens, porém é raro, representando apenas $1 \%$ do total de casos da doença ${ }^{1}$.

As estimativas da incidência de câncer de mama para o ano de 2019, no Brasil, foram de 59.700 casos novos, o que representou 29,5\% dos cânceres em mulheres, excetuando-se o câncer de pele não melanoma. Em 2016, 16.069 mortes de mulheres por câncer de mama ocorreram no nosso país ${ }^{2}$, constituindo um sério problema de saúde pública ${ }^{3}$.

O câncer de mama é uma doença multifatorial, ou seja, não tem somente uma causa. A idade é um dos mais importantes fatores de risco para a doença (cerca de quatro em cada cinco casos ocorrem após os 50 anos de idade). Outros fatores que aumentam o risco da doença são: menstruação precoce, menopausa tardia, uso de contraceptivos orais e terapia de reposição hormonal pós menopausa, sobrepeso/ obesidade, consumo excessivo de álcool, inatividade física e casos de câncer de mama na família (histórico genético) ${ }^{4,5}$.

Os principais sinais e sintomas de câncer de mama são nódulo na mama e/ou axila, dor mamária e alterações da pele que recobrem a mama, como abaulamentos ou retrações com aspecto semelhante à casca de laranja ${ }^{6}$. Cerca de $30 \%$ dos cânceres de mama podem ser evitados com a adoção de hábitos saudáveis como: prática de atividade física, alimentar-se de forma saudável, manter o peso corporal equilibrado ${ }^{7}$.

Entretanto, já existe tratamento para o câncer de mama e o Ministério da Saúde oferece atendimento por meio do Sistema Único de Saúde (SUS) ${ }^{1}$. Ele depende de determinados fatores, tais como: qualidade da assistência prestada ao paciente, situação clínica e psicoemocional, tipo histológico e grau de diferenciação do tumor, extensão da doença e sensibilidade à terapêutica. Cirurgia, radioterapia, quimioterapia, hormonioterapia e terapia biológica são os tratamentos normalmente utilizados quer seja de forma isolada ou combinada ${ }^{5}$.

Cordeiro e Fortes $^{5}$ destacam a necessidade de uma especial atenção das políticas públicas de saúde direcionadas às ações de rastreamento e detecção precoce do câncer de mama em detrimento do elevado risco nutricional e de morbimortalidade. O Instituto Nacional de Câncer José Alencar Gomes da Silva (INCA) ${ }^{4}$ preconiza a realização do exame clínico de mama a partir dos 40 anos de idade; da mamografia, com intervalo máximo de dois anos, após os 50 anos; da combinação dos dois exames anualmente, a partir dos 35 anos, para os grupos com risco elevado.

O autoexame da mama também é utilizado como uma forma de prevenção e diagnóstico precoce. Caracteriza-se como um processo simples e indolor que auxilia na detecção do câncer em seu estágio inicial que pode, inclusive, aparecer na forma de pequenos nódulos mamários. A realização correta do autoexame ocorre uma vez ao mês, durante a segunda semana após a menstruação. A descoberta de nódulos nas mamas propicia a procura de um especialista no intuito de evitar maiores danos, facilitar a terapêutica e, possivelmente, a cura ${ }^{8}$.

Devido à necessidade de maiores esclarecimentos da população sobre a prevenção e o diagnóstico precoce do câncer de mama, o objetivo deste estudo foi elaborar um boletim informativo para a população sobre a importância da prevenção e do diagnóstico precoce por meio do autoexame das mamas. 


\section{MÉTODOS}

A execução deste boletim informativo deu-se em três etapas: duas teóricas e uma prática. Na primeira etapa teórica, fez-se um estudo exploratório por meio de uma revisão narrativa da literatura, promovendo ou aprimorando o embasamento teórico necessário para o desenvolvimento do produto. Analisaram-se dados a partir das principais bases de dados referentes ao câncer de mama, a plataforma online do Ministério da Saúde e do Instituto Nacional de Câncer (INCA), delimitação dos dispositivos utilizados e definição dos conteúdos a serem abordados no Boletim.

A segunda etapa teórica baseou-se na análise de artigos científicos indexados em PubMed/Medline (Literatura Internacional em Ciências da Saúde), Lilacs (Literatura LatinoAmericana e do Caribe em Ciências da Saúde) e SciELO (Scientific Electronic Library Online), sendo os critérios de inclusão, artigos em sua íntegra, com ênfase nos últimos 5 anos (20162020), nas línguas português, inglês e espanhol e que abordassem o tema prevenção e autoexame no câncer de mama. Foram utilizados os descritores: "neoplasias da mama", "autoexame", "prevenção de doenças" e "diagnóstico", e o conectivo AND. Os critérios de exclusão foram teses, dissertações, monografias e artigos que não tratavam especificamente do tema.

Durante a etapa prática, foram geradas alternativas para a produção, tipografia e os possíveis layouts a serem utilizados no documento. Foi sugerida a construção de um texto simples, em formato de boletim explicativo e informativo, visando desenvolver a informação para todo público alvo. O programa utilizado para realização foi o Microsoft Publisher, é um programa do pacote Microsoft Office, que é usado para diagramação eletrônica, como elaboração de layouts com textos, gráficos, fotografias e outros elementos.

\section{RESULTADOS E DISCUSSÃO}

\section{Análise dos artigos}

Foram encontrados 1 (2,6\%) artigo na base PubMed, 23 (60,5\%) na Lilacs e 14 (36,8\%) na SciELO, sendo excluído um total de $34(89,5 \%)$ que não atendiam aos critérios de elegibilidade (Figura 1).
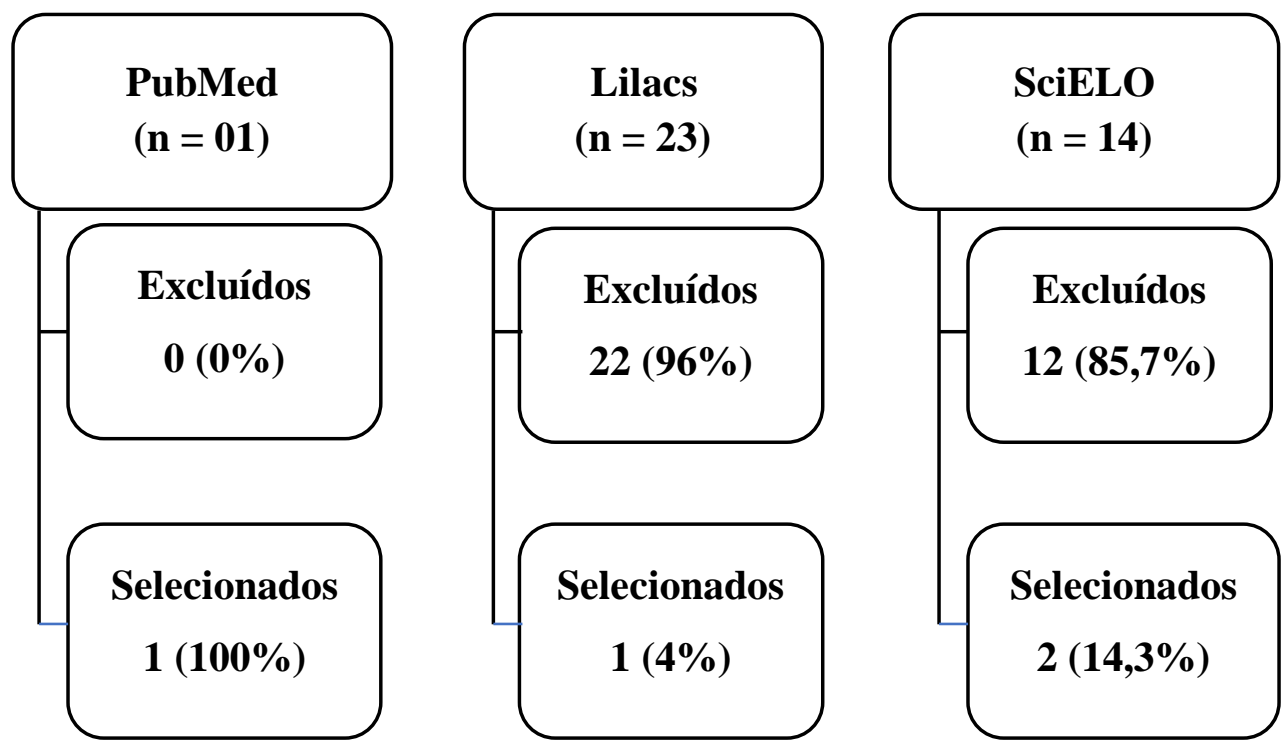
Figura 1 - Distribuição do número de artigos sobre câncer de mama publicados de 2016 a 2020 e disponíveis nas bases de dados Lilacs, SciELO e PubMed, após determinados critérios de seleção.

Ao analisarmos os artigos incluídos nesta revisão narrativa, constatou-se um predomínio de artigos no ano de 2018 com 3 (75\%) estudos, seguido por 2019, com 1 (25\%). (Figura 2).

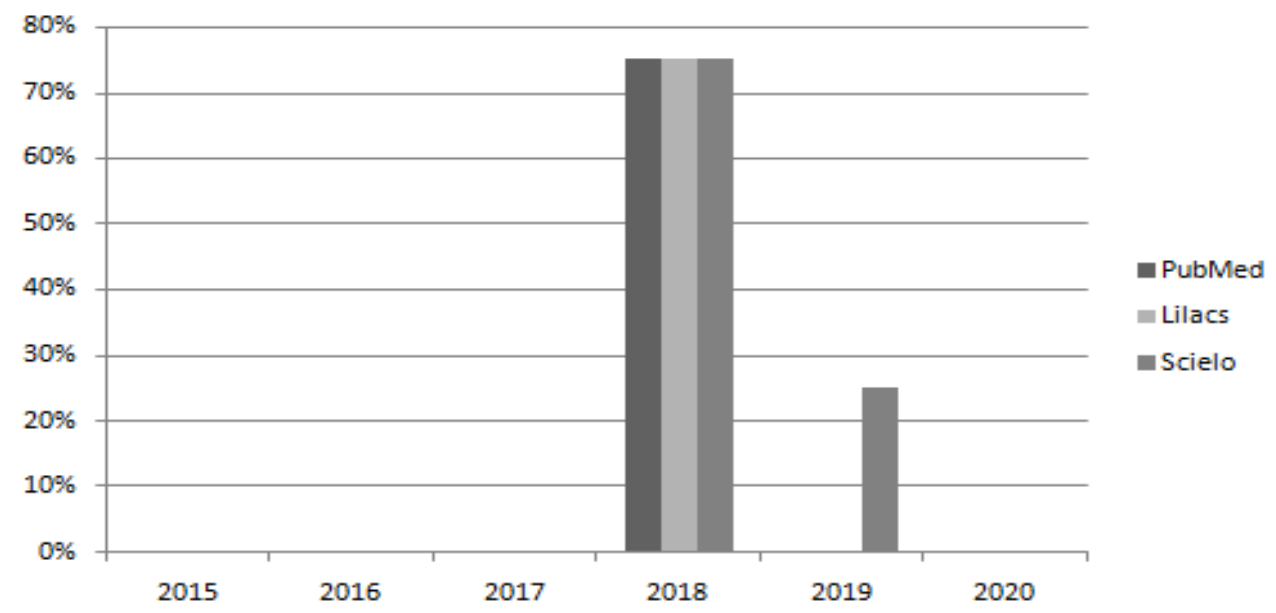

Figura 2 -Distribuição de artigos elegíveis por ano (\%) de publicação nas bases de dados Lilacs, SciELO e PubMed, após determinados critérios de seleção.

\section{Análise do conteúdo}

O câncer de mama é o segundo tipo de neoplasia maligna mais prevalente no mundo e o mais comum entre o sexo feminino, e a sua incidência vem aumentando progressivamente ao longo dos anos. Estimativas apontam que entre os anos de 2016 e 2017 houve uma incidência de, aproximadamente, 600 mil pessoas com câncer de mama, sendo que em 2017 constatou-se uma taxa de incidência bruta de 56,2 casos por cada 100.000 habitantes. Já, em 2018 cerca de 60.000 novos casos de câncer de mama foram estimados ${ }^{3}$. Para 2020, o INCA prevê uma estimativa de 66.280 novos casos de câncer de mama feminina, com 17.572 mortes. É importante, portanto, a realização do autoexame para que haja um rápido diagnóstico e tratamento, evitando assim um avanço da doença.

O câncer é uma doença crônica e degenerativa que possui evolução progressiva cuja etiologia geralmente está associada a uma série de fatores de risco ${ }^{12}$. Os principais fatores de risco para o câncer de mama são: sexo feminino, envelhecimento, gravidez em idade tardia, menarca precoce, menopausa após 55 anos de idade, ciclos menstruais curtos, história familiar de câncer de mama e fatores ambientais ${ }^{6}$. Como essa informação é de suma importância, ela 
foi considerada na elaboração do Boletim informativo, visto que a identificação dos fatores de risco para o desenvolvimento do câncer de mama constitui uma medida imprescindível para a prevenção e o auxílio ao diagnóstico precoce, além de proporcionar maior segurança terapêutica e, em consequência, melhora da qualidade de vida.

Até o momento, inúmeros avanços foram feitos nos estudos clínicos e teóricos do câncer de mama, as formas mais atuais de prevenção são a realização do autoexame, alimentação saudável, atividade física regular, manter o peso corporal adequado, evitar o consumo de álcool e realizar exames periodicamente ${ }^{11}$.

Seabra et al. ${ }^{10}$ estudaram 211 mulheres com câncer de mama a respeito de seus cuidados antes e após o diagnóstico e sua percepção da doença, riscos e autocuidado. Observou-se que $63(29,8 \%)$ das 211 mulheres não realizavam o autoexame, e 43 (20,4\%) realizavam de forma rara, o que mostra que o número de mulheres que seguia para a mamografia de forma mais rápida era bastante reduzido. Os autores defendem que o método mais acurado para detecção do câncer a partir de um nódulo ou massa na mama é a mamografia, que deve ser realizada uma vez ao ano para mulheres acima de 45 anos de idade e uma vez a cada dois anos para mulheres acima de 54 anos.

As formas de diagnóstico do câncer de mama mais comumente utilizadas são os exames de imagem: ultrassonografia e mamografia. A mamografia, padrão ouro na detecção e diagnóstico do câncer, deve ser realizada uma vez ao ano para mulheres entre 45-54 anos, e a cada dois anos para mulheres acima desta faixa etária. ${ }^{10}$ A mesma deve ser realizada em caso de nódulos mamários ou qualquer alteração na percepção das mamas.

O exame das mamas, realizado pela própria mulher, ajuda no conhecimento do próprio corpo, entretanto, esse exame não substitui o exame clínico das mamas, como também a mamografia. Caso a mulher observe alguma alteração deve procurar imediatamente o serviço de saúde mais próximo de sua residência ${ }^{9}$.

O autoexame é importante para que haja uma detecção precoce de qualquer anormalidade nas mamas, como nódulos, texturas diferentes, vermelhidão ou manchas, para que seja possível a realização de um exame diagnóstico precoce e eficiente e assim seja possível um tratamento adequado e em tempo hábil na tentativa de diminuir a mortalidade.

Dentre os sinais e sintomas que devem servir de alerta no autoexame estão: nódulo, fixo e geralmente indolor; pele da mama avermelhada, retraída, ou com aspecto em "casca de laranja", alterações no mamilo, pequenos nódulos na axila ou pescoço, saída espontânea de líquido anormal dos mamilos, prurido, dor, inversão do mamilo e/ou descamação ou ulceração do mamilo ${ }^{1,6}$ como descrito no boletim.

Observando o acima descrito, é importante lembrar que uma detecção precoce da doença pode levar a uma taxa de sobrevida muito maior, com um tratamento mais efetivo e aumentando a qualidade de vida da mulher, o que torna de grande importância o autoexame e o diagnóstico precoces. . $^{1,6}$

É fundamental destacar que a proposta deste Boletim informativo faz parte de um projeto desenvolvido pelo Programa de Residência Multiprofissional em Atenção ao Câncer da ESCS/FEPECS/SES-DF. Os projetos realizados por esse setor dão suporte à saúde e ao bemestar da população por meio de informações a respeito do câncer de mama e sobre a prevenção com a realização do autoexame. Espera-se que a confecção desse material informativo traga subsídios para o melhor entendimento sobre a doença, assim como maior visibilidade sobre os 
benefícios de realizar o autoexame da mama, fornecendo informações aos profissionais de saúde e à população em geral. Além de os resultados que poderão nortear os profissionais que utilizam o método, essas instruções servirão de orientações na prática clínica e beneficiarão a comunidade. Logo, espera-se que haja impacto social no intuito de desmistificar o câncer de mama, promover a sua prevenção e o diagnóstico precoce, com consequente eficácia no tratamento e melhora do prognóstico clínico.

Considerando a relevância do tema e as principais informações obtidas na base de dados do Instituto Nacional de Câncer, confeccionou-se o Boletim informativo conforme a seguir: 


\section{CÂNCER DE MAMA}

Boletim Informativo

Câncer de Mama
câncer de mama é uma doença
causada pela multiplicação
desordenada de células da mama. Esse
processo gera células anormais que se
multiplicam, formando um tumor. é o
mais incidente nas mulheres,
excetuando-se os tumores de pele não
melanoma. Para o Brasil, estimam-se
que 66.280 casos novos de câncer de
mama, para cada ano do triênio 2020
2022. Esse valor corresponde a um
risco estimado de 61,61 casos novos a
cada 100 mil mulheres.

Taxas de mortalidade por câncer de mama feminina, específicas por faixas etárias, por 100.000 mulheres. Brasil,
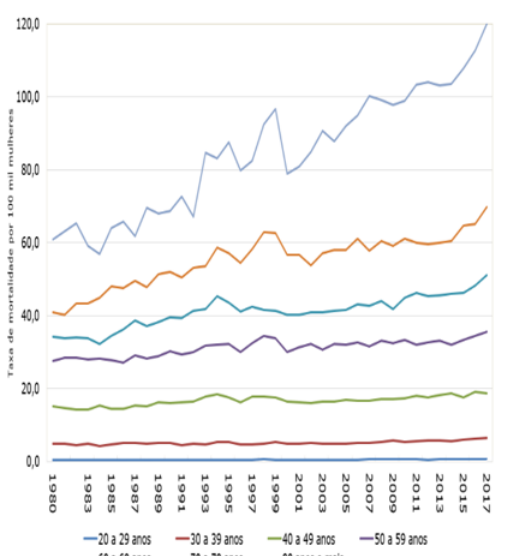

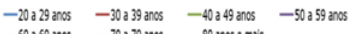

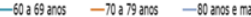

Fonte: Adaptado do Atlas de Mortalidade por Câncer INCA, 2020 . Dados tabulados em 01/06/20, sujeitos revisắo

Assim como a incidência, a mortalidade por câncer de mama é maior em idades mais avançadas. No período analisado, tendência ascendente é observada em algumas faixas etárias, sendo mais expressiva em mulheres de 80 anos e mais.

\section{DIAGNÓSTICO}

Para a investigação, além do exame clínico das mamas, exames de imagem podem ser recomendados, como mamografia, ultrassonografia ou ressonância magnética. A confirmação diagnóstica só é feita, porém, por meio da biópsia.

Fonte: adaptado câncer de mama. INSTITUTO NACIONAL DE CANCER. Ministério
da Saúde, 2020 .

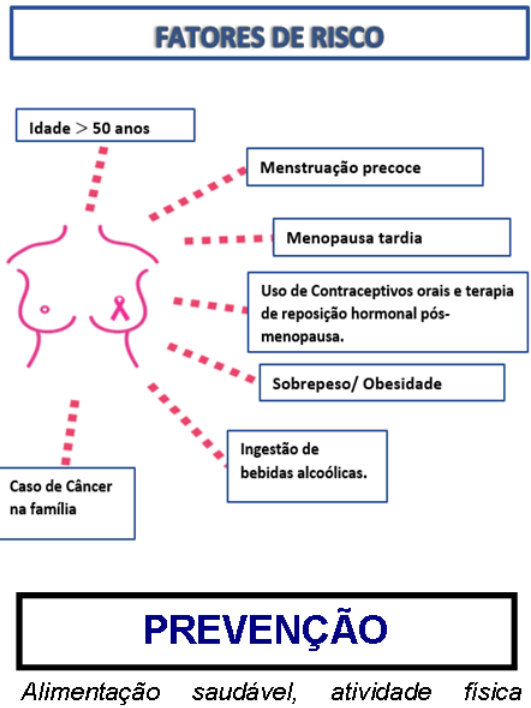

regular, manter o peso comoral adequado, evitar ou reduzir a ingestão de bebida alcoólica é possivel reduzir em até $28 \%$ o risco de desenvolver câncer de mama. 0 Autoexame também fator protetor.

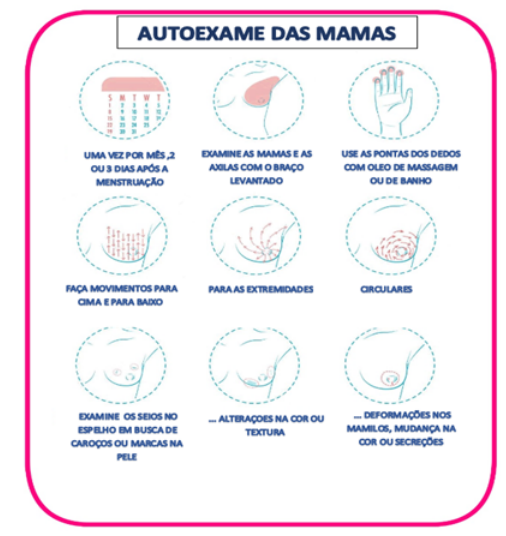

SINAIS E SINTOMAS

- Nódulo (caroço), fixo e geralmente indolor: é a principal manifestação da doença, estando presente em cerca de $90 \%$ dos casos quando o câncer é percebido pela própria muIher

- Pele da mama avermelhada, retraída ou parecida com casca de laranja

- Alterações no bico do peito (mamilo)

- Pequenos nódulos nas axilas ou no pescoço.

- Saída espontânea de líquido anormal pelos mamilos 


\section{CONCLUSÕES}

É evidente que o câncer de mama afeta a vida de mulheres brasileiras, é uma das principais causas de morte desse grupo. A melhor forma de prevenção e detecção precoce é o autoexame das mamas, visto que determinados sinais (abaulamentos, retrações e secreção nos mamilos, vermelhidão, nódulos, dentre outros) podem ser detectados.

No Brasil, a detecção e diagnóstico do câncer de mama ainda se dá de forma tardia. Logo, para que mais mulheres tenham acesso fácil a este método, de forma explicativa e de rápida leitura, foi criado este boletim.

\section{REFERÊNCIAS}

1. Brasil. Instituto Nacional de Câncer José Alencar Gomes da Silva. Câncer de mama. 2020. Rio de Janeiro, INCA. Disponível em: https://www.inca.gov.br/tipos-decancer/cancer-de-mama. Acesso em: 03 de junho de 2020.

2. Brasil. Instituto Nacional de Câncer José Alencar Gomes da Silva. A situação do câncer de mama no Brasil: síntese de dados dos sistemas de informação. / Instituto Nacional de Câncer José Alencar Gomes da Silva. - Rio de Janeiro: INCA, 2019.Disponível em: https://www.inca.gov.br/sites/ufu.sti.inca.local/files/media/document/a_situacao_ca_ mama_brasil_2019.pdf. Acesso em: 15 de junho de 2020.

3. Fortes RC, Haack A, Frazão ERP. Qualidade de vida de mulheres com câncer de mama: uma revisão integrativa. Com. Ciências Saúde. 2018; 29(4):218-225.

4. Brasil. Instituto Nacional de Câncer José Alencar Gomes da Silva. Recomendações do INCA para reduzir a mortalidade por câncer de mama no Brasil. 2017. Rio de Janeiro; INCA. Disponível em:https://www.inca.gov.br/sites/ufu.sti.inca.local/files//media/document/recomendac oes-cancer-de-mama-inca-2017_0.pdf. Acesso em: 03 de junho de 2020.

5. Cordeiro ALO, Fortes RC. Estado nutricional e necessidade de intervenção nutricional em mulheres com câncer de mama em tratamento quimioterápico. Arq. Catarin Med. 2015; 44(4): 96-108.

6. Brasil. Ministério da Saúde. Instituto Nacional de Câncer José Alencar Gomes da Silva. Falando sobre câncer de mama. Rio de Janeiro: INCA; 2002. Disponível em: http://www.saude.pb.gov.br/web_data/saude/cancer/aula11.pdf. Acesso em: 02 de junho de 2020.

7. Brasil. Instituto Nacional de Câncer José Alencar Gomes da Silva. Detecção precoce do câncer de mama. 2015. Rio de Janeiro; INCA. Disponível em: http://www.saude.pr.gov.br/arquivos/File/Deteccao_precoce_CANCER_MAMA_IN CA.pdf. Acesso em: 03 de junho de 2020.

8. Müller MC, Frasson A, Kieling C, Hoffmann FS, Fleck P, Zogbi H et al. A prática do auto-exame das mamas em mulheres de uma comunidade universitária. Psico-USF 2005; 10(2):185-190.

9. Meneses-Echávez JF, Martínez-Torres J, Chavarriaga-Ríos MC, González-Ruiz K, Vinaccia-Alpi S, Rincón-Castro AD et al. Creencias y conocimientos relacionados a la práctica del autoexamen de mama en mujeres universitarias de Colombia: Un estudio descriptivo. Rev. chil. obstet. ginecol. 2018; 83(2): 120-129. 
10. Seabra CR., Armiliato MJ., Souza LV; Otaran P., Peuker ACWB et al. Breast Cancer prevention: what women think about the illness, its risks and self-care. Rev Psico 2018; 49(3): 266-273.

11. Sun YS, Zhao Z, Yang ZN, et al. Fatores de risco e prevenção de câncer de mama. Int J Biol Sci . 2017; 13(11): 1387-1397.

12. Migowski A, Silva GA, Dias MBK, Diz MDPE, Sant'Ana DR, Nadanovsky P. Diretrizes para detecção precoce do câncer de mama no Brasil. II - Novas recomendações nacionais, principais evidências e controvérsias. Cad. Saúde Pública 2018; 34(6): 1-16. 